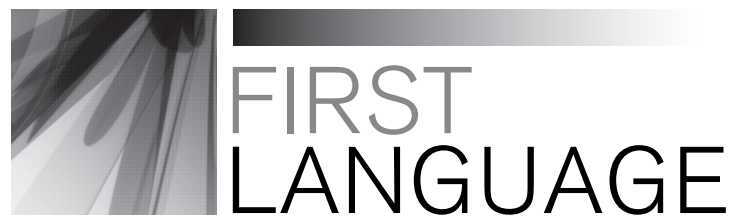

BOOK REVIEW

Copyright (C) The Author(s), 2009. Reprints and permissions: http://www.sagepub.co.uk/journalsPermissions.nav http://fla.sagepub.com Vol 29(3): 340-344 (200908)

DOI: $10.1177 / 0142723709105318$

\title{
The bilingual child: Early development and language contact
}

By Virginia Yip \& Stephen Matthews (Cambridge, UK: Cambridge University Press, 2007). Pp. xxii + 295. ISBN 978-0-52183-617-3 (Hbk), 978-0-52154-476-4 (Pbk).

Yip and Matthews' latest book makes a welcome new contribution to an expanding body of literature on first language acquisition in bilingual contexts. Their starting point is the now well-established view that the bilingual child's languages constitute separate systems. The past decade has witnessed an intense debate over whether the two linguistic systems, although separate in principle, may nevertheless interact. Yip and Matthews provide substantial empirical evidence in favour of this view, arguing that bilingual children develop differently from monolinguals. Moreover, they show that many developmental phenomena in bilingual data are paralleled by similar features in contact varieties, such as Singapore Colloquial English (SCE), and they argue that bilingual acquisition can have the effect of establishing substrate influence in a contact language. In particular, mutual influence occurring in bilingual development - so is the hypothesis - provides one mechanism by which two languages in contact can converge.

Chapter 1 introduces some fundamental questions, specifically: (1) How does bilingual development differ from acquisition of the same two languages by monolingual children? (2) Do the two languages develop independently or is there evidence for transfer or cross-linguistic influence and in the latter case, what factors determine its direction? (3) What do the linguistic features of bilingual children's two developing languages reveal about the processes of language contact and language acquisition?

Chapter 2 provides the theoretical framework, introducing different forms of bilingualism and discussing the relationship between bilingual first language acquisition (BFLA) and early second language acquisition. Correctly, the authors bring the logical problem of language acquisition to bear on BFLA research, claiming that success in attaining bilingual competence strengthens the poverty-of-stimulus argument, 'since the deficiency of input is more acute and the success all the more remarkable [compared to monolingual acquisition]'. The chapter provides a summary of major findings in BFLA research over the past few decades and introduces key 
concepts, such as language differentiation, language dominance, cross-linguistic influence, input ambiguity, vulnerable domains and the relationship between bilingual development and language contact. While spelling out the necessity of having a theoretical model, the authors combine insights from both generative and functional approaches.

Chapter 3 begins with an overview of different data collection methods in BFLA studies, taking note of their respective pros and cons, and then introduces the sizeable Hong Kong Bilingual Child Language Corpus as the book's empirical foundation. The corpus covers naturalistic data from six children (recorded for between 16 and 21 consecutive months) growing up in binational families in Hong Kong with simultaneous exposure to Cantonese and English from birth, three of them the authors' own. Additional diary data (covering longer time spans) are used in the discussion of infrequent structures. Criticism with respect to the selectivity of diary data is effectively respected by combining them with naturalistic data. Conveniently, the corpus is publicly available through the Child Language Data Exchange System (CHILDES) (MacWhinney, 2000). Furthermore, the chapter outlines recording, transcription, and tagging procedures and explains how the children's dominant language was established. MLU is given the greatest weight; further dominance measures discussed are language preference and silent periods. Codemixing is explicitly not considered as an indicator of dominance.

Chapter 4 demonstrates syntactic transfer from Cantonese in the acquisition of English wh-phrases. Four of the Cantonese-dominant children are shown to go through a wh- in situ stage in English at which they do not move the question word. It is convincingly argued that language dominance has an impact on influence, as wh- in situ questions in English are more frequent with increasing dominance in Cantonese. While the quantity of wh-in situ questions coincides with dominance, a more fine-grained qualitative analysis shows that developmental asynchronies occur independently of language dominance: all bilingual children (including English-dominant Charlotte) acquire why-questions earlier in Cantonese than in English and thereby mirror the asynchronies between English and Cantonese monolinguals. At the same time, all bilinguals use English why-questions about five months earlier in English than their monolinguals peers - a fact attributed to positive influence from Cantonese.

Chapter 5 is concerned will null objects. While these are also found in monolingual English children, bilingual children exhibit a prolonged null object stage vis-à-vis monolinguals due to influence from Cantonese, which licenses null topics. Moreover, the 'wrong' analysis for object use in English attributed to the learner is claimed to be reinforced by ambiguities arising from English verbs, e.g., eat and read, as these can appear with a null object or receive a generic interpretation (but see later). Also the phenomenon of an extended object omission period in English supports the role of language dominance, as null objects are used most extensively by Cantonese-dominant learners.

Chapter 6 shows children's use of prenominal relative clauses in English to be attributable to transfer from Cantonese. Once again, transfer is argued to be driven by language dominance and ambiguities in English arising from prenominal modification with modifiers other than relative clauses. 
Interestingly, all cases of influence discussed in Chapters 4-6 are paralleled by Chinese-influenced structures in SCE. This is cited in support of the authors' claim that substrate influence may become established in a contact language through bilingual acquisition.

While Chapters 4 through 6 focus the development in English under the impact of Cantonese - the dominant language of most children studied - Chapter 7 shows the opposite case: despite being stronger, Cantonese demonstrably can be subject to influence as well. In locative prepositional phrases with hai2 'at', all bilingual children use postverbal PPs in Cantonese, suggesting influence from English, while they never use preverbal PPs in English. In double object constructions with bei2 'give', they produce V(erb)-R(ecipient)-T(heme) in Cantonese, which corresponds to the English order, but never the Cantonese V-T-R order in English. Section 7.3 on particle constructions discusses bidirectional influence, which is argued to arise from variable input in both languages (rather than only one).

Chapter 8 compares grammaticalization in the context of language contact to similar processes in the development of bilingual individuals. The discussion is based on particularly salient cases in the bilingual data, including the emergence of already as an aspect marker, the development of grammatical functions of the verb give, and the use of one as nominalizer. The bilingual children's development replicates the process by which certain grammatical patterns have emerged in SCE through the substrate influence of Chinese. It is argued that the bilingual speakers' innovative use of old items in new grammatical functions (so-called cases of 'idiogrammaticalization') logically precedes contact-induced grammaticalization. Such innovations may help children bridge temporary gaps arising whenever one language dominates while structures in the weaker language still have to be acquired. Hence, BFLA is a possible route for substrate influence in the development of contact languages.

Chapter 9 provides final concluding remarks.

Certainly not the first book on BFLA, this one is to be welcomed for providing an up-to-date overview of theoretical and empirical issues in BFLA, illustrated with rich and abundant data on a typologically distinct language pair. The book is highly readable and informative, providing a valuable methodological resource, despite its rather narrow focus on syntax and simultaneous child bilingualism. Though largely drawing on data from English and Cantonese, the authors' methodology is easily applicable to the study of further language pairs. Conveniently for the reader, individual chapters constitute fairly self-contained units. Since the authors adopt insights from both UG and usage-based frameworks, the book will be of interest to researchers in both fields. Throughout, the postulated link between BFLA and the development of contact language on the theoretical level is matched by empirical examples. However, while much space has been dedicated to acquisition data, Chapter 8 on contact-induced grammaticalization is likely too concise for readers without any background in this area.

The authors provide clear evidence for the thesis that the direction of influence is not random. In fact, in the majority of cases, the stronger language affects the weaker one. Additionally, influence can be determined by input ambiguities and developmental asynchronies, i.e., cases where certain structures are acquired earlier 
in one language than the corresponding structures in the other language. The neutrality of the presentation is refreshing and, generally, to be commended. With respect to some data patterns, however, a more critical discussion of the factors driving influence might have been possible. One example is the use of null objects in English. Similar to Müller and Hulk (2001) in their French and Italian data, Yip and Matthews relate the extended null object stage in English to the topic-drop property of the contact language. However, Pérez-Leroux, Pirvalescu, and Roberge (2009) found a delay in the French of French-English bilinguals, although English is not a topic-drop language. First, this suggests that the topic-drop property of the contact language is not crucial for influence to occur. Second, it raises the question why influence is not bidirectional in Cantonese-English bilinguals. Since bidirectional influence is considered possible in principle (see participle constructions, 7.3.), one could ask why object use is ambiguous only in English but not in Cantonese: in Cantonese, too, one and the same verb can be found with a lexical object or without an object.

This book shows that in BFLA research we have strong intuitions about the forces driving cross-linguistic influence (and, possibly, as a consequence, the development of contact languages), but it also reveals that BFLA research is still a far cry from providing a model that is able to account for the necessary and sufficient conditions by which influence and its direction can be predicted. First, some children show influence when it is predicted, but others do not. Second, since there are cases in which the weaker language influences the stronger one (see sections 7.1-7.3), language dominance cannot be necessary for influence to occur. The authors attribute such unexpected weaker-to-stronger language transfers to 'vulnerability', which - so the data suggest - is not related to the CP (unlike in Platzack's [2001] original pro-posal). However, in the end the notion of vulnerability remains rather vague. It is noted that 'other factors [other than dominance] must be involved, including in particular properties of the input such as input ambiguity' (p. 190). But since input ambiguities are also held responsible for the stronger-to-weaker language influence in Chapters 4-6, 'vulnerability' has the flavour of a post-hoc descriptive term for cases where influence takes an unexpected path. It is thus left for future work to provide an independent account of 'vulnerability'.

Overall, the combination of typologically distinct languages studied makes this book an especially important contribution to the study of BFLA and contact languages. It will be of great interest to researchers and it is suitable for coursework at both undergraduate and graduate level, although the focus on syntax may be too narrow to cover an entire course on bilingual language acquisition.

Tanja Kupisch University of Hamburg

\section{REFERENCES}

MacWhinney, B. (2000) The CHILDES Project: Tools for analyzing talk, transcription format and programs. Mahwah, NJ: Erlbaum. 
Müller, N., \& Hulk, A. (2001). Cross-linguistic influence in bilingual language acquisition: Italian and French as recipient languages. Bilingualism: Language and Cognition, 4, 1-21. Pérez-Leroux, A., Pirvulescu, M., \& Roberge, I. (2009). Bilingualism as a window into the language faculty: The acquisition of objects in French-speaking children in bilingual and monolingual contexts. Bilingualism: Language and Cognition, 12(1), 97-112.

Platzack, C. (2001). The vulnerable C-domain. Brain and Language, 77, 364-377. 